\title{
Quasicontinuum Models of Interfacial Structure and Deformation
}

\author{
V. B. Shenoy, ${ }^{1}$ R. Miller, ${ }^{1}$ E. B. Tadmor, ${ }^{2}$ R. Phillips, ${ }^{1}$ and M. Ortiz ${ }^{3}$ \\ ${ }^{1}$ Division of Engineering, Brown University, Providence, Rhode Island 02912 \\ ${ }^{2}$ Department of Physics, Harvard University, Cambridge, Massachusetts 02138 \\ ${ }^{3}$ Graduate Aeronautical Laboratories, California Institute of Technology, Pasadena, California 91125
}

(Received 10 July 1997)

\begin{abstract}
Microscopic models of the interaction between grain boundaries (GBs) and both dislocations and cracks are of importance in understanding the role of microstructure in altering the mechanical properties of a material. A recently developed mixed atomistic and continuum method is reformulated to allow for the examination of the interactions between GBs, dislocations, and cracks. These calculations elucidate plausible microscopic mechanisms for these defect interactions and allow for the quantitative evaluation of critical parameters such as the force needed to induce GB migration. [S0031-9007(97)05134-X]

PACS numbers: 62.20.- $\mathrm{x}, 68.45 . \mathrm{Nj}, 81.40 . \mathrm{Lm}$
\end{abstract}

With the continuing development of more accurate, less expensive models for atomistic interactions and expansion of computational resources, there is growing interest in the modeling of materials from fundamental principles rather than phenomenological approaches. An outstanding problem in this regard is the role of microstructure in determining material properties. The influence of microstructure (e.g., grain size and shape) on the mechanical properties of materials is clearly revealed, for example, in the yield strength and the fracture toughness [1]. A first step in the microscopic determination of the role of microstructure in governing such properties is the elucidation of plausible mechanisms whereby dislocations and cracks, the primary agents of permanent deformation, interact with the boundaries that make up that microstructure. One of the key challenges posed by such calculations is the simultaneous operation of multiple scales in the problem requiring alternative simulation schemes.

In this Letter, we present a reformulation of one such method for treating multiple scales and demonstrate its application to two examples: The interaction of lattice dislocations with grain boundaries (GBs) and the interaction of cracks with GBs.

The quasicontinuum method [2], a mixed atomisticcontinuum formulation, is based on a finite element discretization of a continuum mechanics variational principle. The finite element method serves as the numerical engine for determining the energy minimizing displacement fields, while atomistic analysis is used to determine the energy of a given configuration. This is in contrast to standard finite element approaches, where the constitutive input is made via phenomenological models. The method is successful in capturing the structure and energetics of dislocations.

In this paper we consider a reformulation of the method that allows for the treatment of interfaces, and show how it allows for the simultaneous treatment of dislocations, material interfaces, and cracks. The key new idea in the formulation is that rather than considering an atomistic scheme for providing constitutive input to a continuum model, which requires the definition of an energy density near the grain boundary, we begin with the recognition that from the microscopic perspective the body may be regarded as a collection of $N$ atoms. The total potential energy of such a collection is given by

$$
\Pi=\sum_{i=1}^{N} E_{i}\left(\mathbf{r}_{1}, \ldots, \mathbf{r}_{N}\right)-\sum_{i=1}^{N} \mathbf{f}_{i} \cdot \mathbf{r}_{i},
$$

where $\mathbf{r}_{i}$ is the position of the atom $i, \mathbf{f}_{i}$ is the external force on that atom, and $E_{i}$ is its energy as would be computed from an atomistic model such as the embedded atom method (EAM) [3] used here. One of the primary objectives in the formulation of the method is to eliminate the redundant atomistic degrees of freedom associated with the regions of the body far from extended defects and hence subject to displacement fields which are slowly varying on the atomic scale. To achieve the requisite degree of freedom reduction, we select $M$ representative atoms from the $N$ atoms $(M \ll N)$, chosen to best represent the energetics of the body, the positions $\mathbf{r}_{\alpha}$ $(\alpha=1, \ldots, M)$ of which serve as the reduced set of degrees of freedom. The body is now divided into disjoint cells such that each cell contains exactly one representative atom. The key energetic approximation is that the energy of all of the atoms in a given cell is the same as that of the cell's representative atom. The positions of the atoms that are not treated explicitly are obtained by interpolating the nodal values of the displacements using a finite element mesh which is constructed with the representative atoms as the nodal points. (One possible implementation of this strategy in two dimensions is to use the Voronoi polygons [4] surround the representative atoms as the cells and the geometric dual of the Voronoi tiling, the Delaunay triangulation [5], as the finite element mesh.)

Given the scheme described above, the approximate potential energy depends only on the positions of the representative atoms $\mathbf{r}_{\alpha}$ and can be written as

$$
\Pi_{\text {reduced }}=\sum_{\alpha=1}^{M} n_{\alpha} \bar{E}_{\alpha}\left(\mathbf{r}_{1}, \ldots, \mathbf{r}_{M}\right)-\sum_{\alpha=1}^{M} n_{\alpha} \overline{\mathbf{f}}_{\alpha} \cdot \mathbf{r}_{\alpha},
$$


where $n_{\alpha}$ is the number of atoms represented by atom $\alpha$, $\bar{E}_{\alpha}$ is the energy of that representative atom, and $\overline{\mathbf{f}}_{\alpha}$ is the effective force acting on the $\alpha$ th representative atom. In practice, $\bar{E}_{\alpha}$ is computed in two different ways. When the representative atom is located in a region undergoing strongly nonuniform deformation, $\bar{E}_{\alpha}$ is computed using the usual atomistic rule in which a given atom is surrounded by its complement of neighbors and the resulting energy per atom is computed. On the other hand, if the representative atom experiences a slowly varying deformation the energy is still computed atomistically, but with the assumption that its environment is distorted according to the local gradients of deformation. The fully atomistic scheme is used in regions undergoing inhomogeneous deformation on the scales comparable to that of the lattice spacing, such as at dislocation cores and the active regions of the grain boundaries (such as those parts that are in the proximity of dislocations). The formulation based on the deformation gradients is used in regions undergoing near-homogeneous deformation. It is also used in the regions of the grain boundaries that are not active. This ensures that the elastic mismatch between the grains is captured correctly in those regions where the atomistic details of the boundary are unimportant.

Another crucial ingredient of the methodology is adaptive remeshing which allows the mesh to be refined/coarsened to capture the essence of the evolving deformation. If, for example, a dislocation approaches a part of a grain boundary in a coarse mesh region, the adaptive meshing strategy will automatically remesh the region around the pertinent part of the boundary to include every atom in the region as a node of the finite element mesh, reducing the calculation to a fully atomistic one. The details of methodology will be described elsewhere. The key outcome gained in the implementation of the strategy described above is the incorporation of the relevant atomistic nonlinearity and nonlocality that allows for the emergence of defects, such as dislocations and cracks, without the attendant singularities that plague linear elastic analyses, or the burden of many redundant atomistic degrees of freedom.

For the purposes of the present paper, the method must successfully reproduce the known static geometric structures of GBs, since it is the incorporation of boundaries that represents a significant departure from earlier studies [2]. As a test of the method, we have examined the structure of a range of GBs in several fcc metals. For the moment, we have confined our attention to symmetric tilt boundaries, using embedded-atom-type potentials. The key quantitative tests of the outcome of these calculations are an appropriate reckoning of (i) the interfacial energy and (ii) the interfacial structure. An indication of the typical energy differences between the quasicontinuum result and the associated direct atomistic calculation is demonstrated by a $\Sigma 5(210) \mathrm{GB}$ in $\mathrm{Au}$, where the energy as obtained by conventional atomistics $\left(676 \mathrm{~mJ} / \mathrm{m}^{2}\right)$ [6] and the method described here $\left(670 \mathrm{~mJ} / \mathrm{m}^{2}\right)$ are in close agreement. Similarly, in all of the cases we have consid- ered [i.e., $\Sigma 5(210)$ in $\mathrm{Al}$ and $\mathrm{Cu}, \Sigma 3(111)$ in $\mathrm{Al}, \Sigma 99(557)$ in $\mathrm{Al}$, and $\Sigma 21(\overline{2} 41)$ in $\mathrm{Al}, \mathrm{Au}$, and $\mathrm{Ni}$, the atomic level geometry at the interface obtained using the quasicontinuum method advocated here was, for practical purposes, identical to that obtained using direct atomistic simulation. The success of these calculations suggests the viability of using these methods in the context of interactions between dislocations, cracks, and GBs.

Despite the existence of useful continuum models of dislocation-GB interactions, it remains a crucial challenge to uncover the microscopic processes that transpire once the dislocation core is in the proximity of a GB. Our earlier work on simulating nanoindentation [7] suggests the possibility of using nanoindentation-induced dislocations to probe the interaction between dislocations and a GB. As a model system, we consider a block oriented such that (111) planes are positioned to allow for the emergence of dislocations which then travel to the $\Sigma 21(\overline{2} 41) \mathrm{GB}$ which waits approximately $200 \AA$ beneath the surface [cf. Fig. 1(a)].

In preparation for this simulation, we must first find the energy minimizing configuration of the $\Sigma 21$ boundary itself. In anticipation of the possibility of slip transmission, this geometry involves two sets of (111) planes belonging to the two grains and terminating at the boundary. Once the equilibrium grain boundary structure is determined, a mesh is constructed such as that shown in Fig. 1(a). The region that is expected to participate in the dislocation-GB interaction is meshed with full atomistic resolution, while in the far fields the mesh is coarser. The model is then loaded using displacement boundary conditions at the indentation surface and, after a critical load level is reached, dislocations are nucleated at the surface.

Because we used a relatively high stacking fault energy associated with the EAM potentials for Al [8], the dislocations nucleated at the free surface are produced as rather closely spaced $(15 \AA)$ Shockley partials. As seen at the left in Fig. 1(b), the Shockley partials have been absorbed at the GB with the creation of a step at the GB and no evidence of slip transmission into the adjacent grain. This geometry can be rationalized on the basis of the underlying displacement shift complete (DSC) lattice [9] associated with this symmetric GB. We find that the lattice dislocation $\frac{a_{0}}{2}[\overline{1} 10]$ can be split into two DSC lattice vectors,

$$
\frac{a_{0}}{2}[\overline{1} 10]=\underbrace{\frac{a_{0}}{14}[\overline{3} \overline{1} \overline{2}]}_{\text {GB-dislocation }}+\underbrace{\frac{a_{0}}{7}[\overline{2} 41]}_{\text {step }},
$$

where $\frac{a_{0}}{14}[\overline{3} \overline{1} \overline{2}]$ is the Burgers vector of a GB dislocation parallel to the GB and $\frac{a_{0}}{7}[\overline{2} 41]$ is the vector associated with the step. The dislocation with the $\frac{a_{0}}{14}[\overline{3} \overline{1} \overline{2}]$ Burgers vector is accompanied by a sliding motion of the grain boundary.

As the load is increased, a second pair of Shockley partials is nucleated, and they are not immediately absorbed into the GB and, consequently, form a pileup (cf. Fig. 1). These dislocations are not absorbed until a much higher 

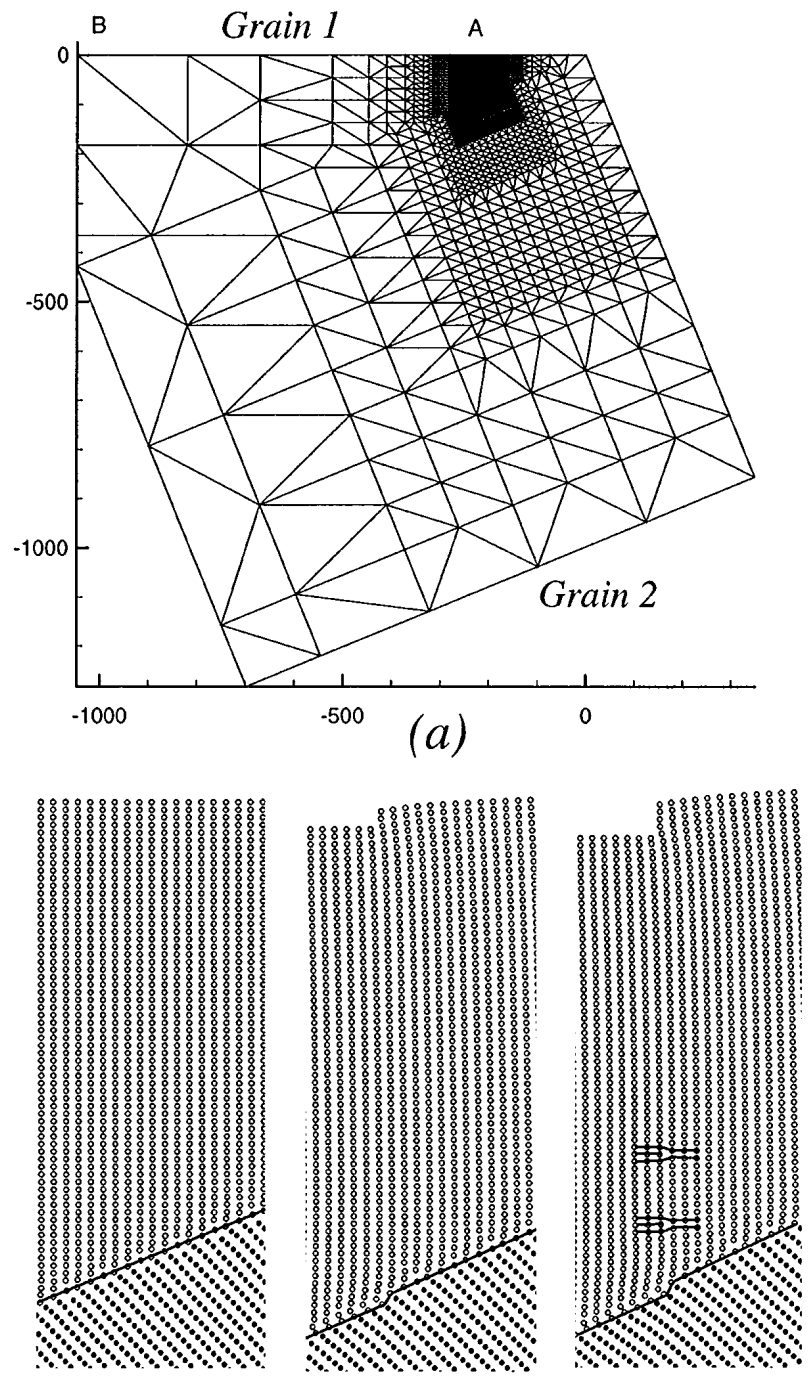

(b)

FIG. 1. (a) Finite element mesh used to model dislocation-GB interaction. The surface marked $A B$ is rigidly indented in order to generate dislocations at $A$ (distance in $\AA$ ). (b) Snapshots of atomic positions at different stages in the deformation history. Absorption of the first pair of dislocations at the GB results in a step, while the second pair form a pileup.

load level is attained. Even after the second set of dislocations is absorbed at the boundary, there is no evidence of slip transmission into the adjacent grain, although the boundary structure itself becomes much less ordered. Although this simulation illustrates the mechanisms involved in the dislocation-GB interaction for this particular boundary, it also raises questions about the general rules governing either absorption or transmission of dislocations at GBs.

As a second example of the synthetic view of extended defects afforded by this method, we consider the interaction between a brittle crack and a GB. The interaction of cracks and interfaces poses a variety of challenging and important problems. One issue that can be considered within the confines of the method presented here is that of the interaction of a crack propagating by cleavage as it impinges upon a GB in its path. The issues that attend the use of the method for considering fracture in general will be presented elsewhere, while here we will note the key elements in carrying out such simulations.

In order to investigate the interaction between an advancing crack and a GB, we consider the $\Sigma 21(\overline{2} 41) \mathrm{GB}$ in fcc nickel. A crack is initiated in one of the grains by removing a single (111) plane, such that the crack tip is located about $2 \mathrm{~nm}$ from the GB. The crack is then loaded by applying the isotropic linear elastic displacement fields for a sharp crack at the mesh boundaries. The load is incrementally increased by scaling the boundary node displacements and allowing the interior nodes to relax to their minimum configuration.

Two snapshots of the solution are shown in Fig. 2. We show the atoms associated with that part of our finite element mesh that is fully refined to the atomic scale in the immediate vicinity of the crack tip. The surrounding mesh, which extends about $300 \mathrm{~nm}$ in each direction, has been removed for clarity. The dots are atomic positions, while the contours reveal displacement jumps across active slip planes, indicating the presence of dislocations. Figure 2(a) shows the configuration after four load steps. The atom labeled "ct" indicates the initial location of the crack tip, and one can see that the crack has begun to propagate towards the GB by cleavage. Light grey slip traces emanating from the GB, such as those labeled "d1" and "d2" show where the stressed GB has emitted dislocations. The dashed line running diagonally through the figure indicates the initial location of the GB which moves as a result of the high stresses in the crack tip region. This motion is accommodated by the structural rearrangement of atoms in the left-hand grain to lattice sites in the righthand grain due to shearing along atomic planes. The solid line through the figure indicates the location of the GB after migration.

In Fig. 2(b), the solution after another few load steps is depicted. Here, the crack has reached the GB and has been blunted when atoms above the plane of the crack again undergo a shearing deformation. This time, how-

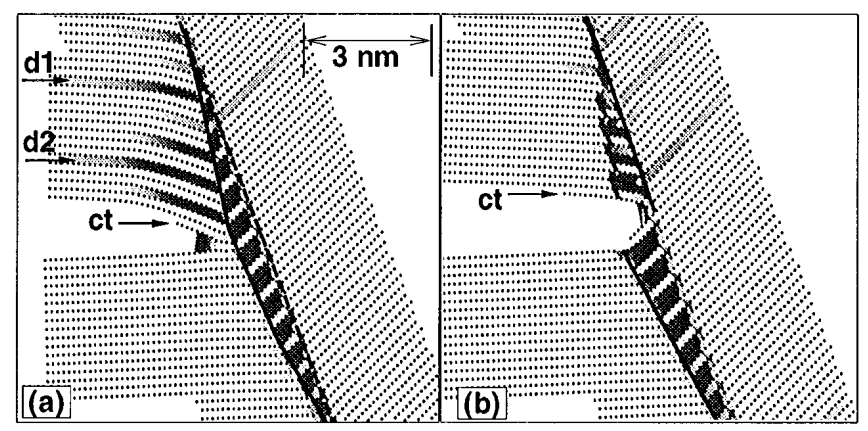

FIG. 2. Crack-GB interaction. Snapshots of crack tip region showing motion of crack tip, dislocations, and GB. d1 and d2 are dislocations emitted from the boundary, and ct represents the initial position of the crack tip. 
ever, the right-hand grain shears to match the structure of the left-hand grain. The two straight solid lines indicate the new location of the GB. The result of this crack blunting is a significant reduction in the stress levels above the crack, and dislocations such as $\mathrm{d} 1$ and $\mathrm{d} 2$ in the first frame have moved back to be reabsorbed by the GB. Further loading of the crack leads to a continued crack blunting due to shearing of atomic planes along the GB. We have studied other GBs [10], where the crack has deflected and continued to propagate along the GB, in contrast to the crack blunting mechanism described here. In particular, for the purposes of comparison, we note that, in the case of a $\Sigma 5(120)$ boundary in the presence of a crack, there is neither dislocation emission from the boundary nor motion of the boundary. Rather, the crack advances to the boundary and ultimately cleaves along it (see [10]).

To understand these results, we turn to continuum mechanics which provides the basis for evaluating the energetic origins of GB migration. Such reasoning asserts that the driving force on an interface is given by the jump in the Eshelby tensor [11] across the interface, with this tensor defined as

$$
P_{i j}=W \delta_{i j}-u_{k, i} \sigma_{k j} .
$$

$W$ is the strain energy density, $u_{i, k}$ is the $k$ th component of displacement, and $\sigma_{k j}$ is the stress tensor. Within the confines of linear elasticity, we have computed the driving force on the interface by using a conventional anisotropic linear elastic constitutive model in conjunction with the standard finite element method to obtain the fields associated with the crack-GB geometry described above. Once these fields are obtained, the resulting driving force may be obtained by computing the jump in the Eshelby tensor across the interface. If we further assume that the GB migration is proportional to the driving force, the driving force profile may be compared directly with the bowed-out geometry as shown in Fig. 3. The linear elastic calculation of the driving force provides a plausible first step in the attempt to understand the stress-induced motion of grain boundaries. The success of the calculation given above makes it of greater interest to uncover the kinetics
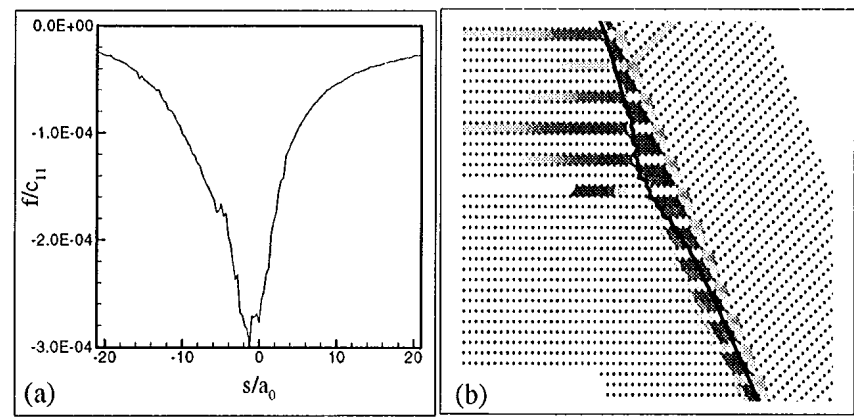

FIG. 3. (a) Driving force (normalized by the elastic constant, $c_{11}$ ) as a function of position $s$ along the GB (normalized by the lattice constant $a_{0}$ ). (b) The same force superimposed on the GB for comparison. of such motion: In particular, what is the relation between the driving force and the boundary velocity?

In this Letter, we have shown how our mixed atomistic and continuum analyses have been adapted to the treatment of interfacial deformation. Such calculations demanded the generalization of the original quasicontinuum formulation to allow for the existence of more than one grain at the same time. As validation of the method, we have computed the structure and energetics of a series of different GBs and found entirely satisfactory correspondence between these calculations and those resulting from direct atomistics. The method was then applied to two distinct problems: the interaction between dislocations and a GB, and the propagation of a crack into a GB. The former revealed the details of the "dislocation-GB chemistry," while calculations on the crack-GB interaction revealed stressinduced GB motion which can be rationalized in terms of the driving force on that interface as implied by the jump in the Eshelby tensor. The advantage of the model presented here over standard atomistic calculations is the significant reduction in the computational effort through careful reduction of the degrees of freedom. For example, the number of degrees of freedom associated with the mesh of Fig. 1(a) is about $10^{4}$, while the same atomistic calculation would have required more than $10^{7}$ degrees of freedom.

We are grateful to C. Briant, R. Clifton, B. Gerberich, P. Hazzledine, S. Kumar, D. Rodney, and A. Schwartzman for discussions, to S. W. Sloan for use of his Delaunay triangulation code, and to M. Daw and S. Foiles for use of their DYNAMO code. We are also grateful to AFOSR who supported this work under Grant No. F49620-95I-0264, the NSF through Grants No. CMS-9414648 and No. DMR-9632524, and the DOE through Grant No. DEFG02-95ER14561. R. M. acknowledges the support of the NSERC.

[1] B. Lawn, Fracture of Brittle Solids (Cambridge University Press, Cambridge, England, 1993), 2nd ed.

[2] E. B. Tadmor, M. Ortiz, and R. Phillips, Philos. Mag. A 73, 1529 (1996).

[3] M.S. Daw and M. I. Baskes, Phys. Rev. Lett. 26, 583 (1971).

[4] A. Okabe, B. Boots, and K. Sugihara, Spatial Tessellations (Wiley, New York, 1992).

[5] S. W. Sloan, Comput. Struct. 47, No. 3, 441-450 (1993).

[6] G. J. Ackland, G. Tichy, V. Vitek, and M. W. Finnis, Philos. Mag. A 56, 735 (1987).

[7] E. B. Tadmor, R. Phillips, and M. Ortiz, Langmuir 12, 4529 (1996).

[8] F. Ercolessi and J. B. Adams, Europhys. Lett. 26, 583 (1994).

[9] A. H. King and D. A. Smith, Acta Crystallogr. Sect. A 36, 335 (1980).

[10] R. Miller, Ph.D. thesis, Brown University (1997).

[11] J. D. Eshelby, J. Elast. 5, 321 (1975). 